\title{
Skeletal Muscle Quality is Associated with Worse Survival After Pancreatoduodenectomy for Periampullary, Nonpancreatic Cancer
}

\author{
L. B. Van Rijssen, $\mathrm{MD}^{1}$, N. C. M. van Huijgevoort, $\mathrm{MD}^{1}$, R. J. S. Coelen, $\mathrm{MD}^{1}$, J. A. Tol, MD, PhD ${ }^{1}$, \\ E. B. Haverkort, MSc, $\mathrm{PhD}^{2}$, C. Y. Nio, $\mathrm{MD}^{3}$, O. R. Busch, MD, PhD ${ }^{1}$, and M. G. Besselink, MD, MSc, PhD ${ }^{1}$ \\ ${ }^{1}$ Department of Surgery, Academic Medical Center, Amsterdam, The Netherlands; ${ }^{2}$ Department of Nutrition and Dietetics, \\ Academic Medical Center, Amsterdam, The Netherlands; ${ }^{3}$ Department of Radiology, Academic Medical Center, \\ Amsterdam, The Netherlands
}

\begin{abstract}
Background. Body composition measures may predict outcomes of cancer surgery. Whereas low muscle mass shown on preoperative computed tomography (CT) scans has been associated with worse outcomes after surgery for pancreatic cancer, less consideration has been given to low muscle attenuation, reflecting poor muscle quality. Studies relating muscle mass and muscle attenuation with outcomes for patients with periampullary, nonpancreatic cancer are lacking.

Methods. Skeletal muscle mass and attenuation were assessed in 166 consecutive patients undergoing pancreatoduodenectomy (PD) for periampullary, nonpancreatic cancer at a single center between 2000 and 2012. The skeletal muscle index (SMI) was calculated from crosssectional muscle area on preoperative CT imaging at the third lumbar vertebra level (L3) and normalized for height. The skeletal muscle attenuation index (MAI) was calculated by measuring the average Hounsfield units of the total muscle area at the L3 level. Overall survival (OS) and the rate of major postoperative complications (Clavien-Dindo $\geq 3$ ) were extracted from prospectively maintained databases.
\end{abstract}

Electronic supplementary material The online version of this article (doi:10.1245/s10434-016-5495-6) contains supplementary material, which is available to authorized users.

(C) The Author(s) 2016. This article is published with open access at Springerlink.com

First Received: 6 April 2016; Published Online: 8 September 2016

M. G. Besselink, MD, MSc, PhD

e-mail: m.g.besselink@amc.uva.nl
Results. Low SMI was present in $78.3 \%$ and low MAI in $48.8 \%$ of the patients. The multivariate analysis showed lymph node metastasis [hazard ratio (HR) 1.8; $95 \%$ confidence interval (CI) 1.1-2.9], microscopic radicality (HR 2.0; $95 \%$ CI 1.2-3.4), and low MAI (HR 2.0; $95 \%$ CI 1.2-3.3), but not low SMI to be significantly associated with decreased OS. Low MAI (HR 1.9; $95 \%$ CI 1.0-3.8) was the only independent risk factor for major postoperative complications.

Conclusion. Skeletal muscle quality, but not muscle mass, predicted survival and major complications after PD for periampullary, nonpancreatic cancer. Preoperative CTderived body composition measures may stratify patients into risk categories and support shared decision making.

Recently, the value of computed tomography (CT)-derived body composition measures for predicting postoperative outcomes has gained interest. For example, loss of skeletal muscle mass shown on preoperative $\mathrm{CT}$ imaging has been associated with worse short- and long-term outcomes after resection of tumors of varying origin. ${ }^{1}$ Also in gastrointestinal and hepato-pancreato-biliary (HPB) surgery, ${ }^{2}$ including surgery for pancreatic cancer, ${ }^{3-6}$ low skeletal muscle mass has been associated with increased morbidity and mortality and worse survival. Less consideration has been given to muscle attenuation shown on $\mathrm{CT}$ imaging, which has been associated with worse survival for patients with solid tumors of respiratory and gastrointestinal origin, for patients with melanoma or metastatic renal cell carcinoma, ${ }^{7-9}$ and recently for patients with pancreatic cancer. ${ }^{4,5,10}$

Low muscle attenuation reflects decreased muscle quality by an accumulation of intramuscular lipid depositions (myosteatosis), and the presence of myosteatosis on 
preoperative CT imaging may be shown through a negative correlation with the amount of intramuscular adipose tissue. ${ }^{1-13}$ Both myosteatosis and loss of muscle mass lead to a decrease in muscle strength. ${ }^{14}$

Studies associating preoperative CT-derived body composition measures of patients having periampullary, nonpancreatic cancer with survival and major postoperative complications do not exist. However, periampullary cancer constitutes about one third of all patients undergoing pancreatoduodenectomy (PD). ${ }^{15}$ The 5-year survival rate after resection of periampullary (distal bile duct, papilla, and duodenum) cancer may reach $50 \%$, twice the rate for pancreatic cancer patients. ${ }^{15,16}$ Mortality rates after $\mathrm{PD}$ are about 1 to $3 \%$ in expert centers, but postoperative morbidity occurs in up to $50 \%$ of patients. ${ }^{15,17}$ Therefore, risk stratification and response prediction before treatment remain of great interest. We assessed the association of skeletal muscle mass and muscle attenuation (quality) with OS and major postoperative complications in patients undergoing PD for periampullary, nonpancreatic cancer.

\section{PATIENTS AND METHODS}

\section{Study Cohort and Data Acquisition}

All patients who underwent PD between 2000 and 2012 for primary papilla of Vater (C24.1), extrahepatic bile duct (C24.0), or duodenal (C17.0) adenocarcinoma were selected from a prospectively maintained database at a single center. Patients with pancreatic ductal adenocarcinoma (C25) were excluded. Topography and morphology were coded according to the international Classification of Diseases for Oncology (ICD-O). The primary outcome was OS, defined as the time between PD and death. Patients were observed until death or 1 September 2015, at which time they were censored. Survival data was obtained from

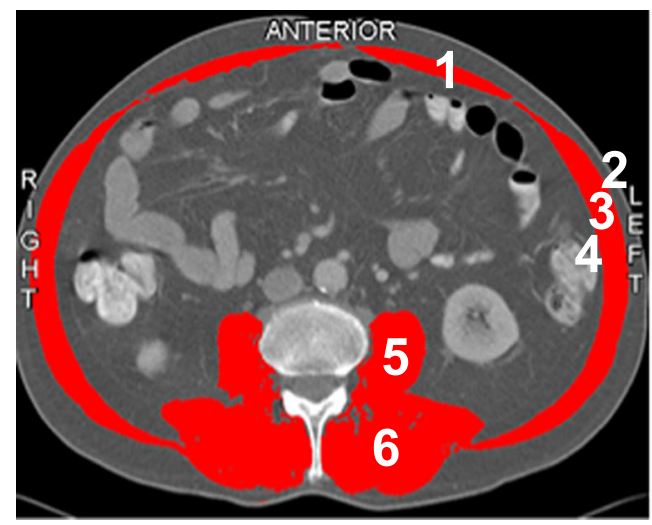

FIG. 1 Computed tomography scans at the third lumbar vertebrae level of two male patients. Right Patient with a low skeletal muscle index (SMI, 56.8) (muscle mass) but a normal muscle attenuation index (MAI, 49.8) (muscle quality). Right Patient with a normal SMI the Municipal Personal Records Database, the central registry for all Dutch inhabitants.

The secondary outcome was the rate of major postoperative complications, defined as any complication classified as Clavien-Dindo grade 3 or higher within 30 days after PD or during admission, whichever was longer. ${ }^{18}$ Overall morbidity was evaluated and consisted of surgical and nonsurgical complications. The incidence of major postoperative pancreatic fistula, postpancreatectomy hemorrhage, and delayed gastric emptying also were noted, all according to the International Study Group of Pancreatic Surgery definitions. ${ }^{17,19,20}$

\section{CT Image Analysis}

The final preoperative CT scan was used to determine skeletal muscle mass, muscle attenuation, and adipose tissue area. Patients were excluded from the analysis if no preoperative CT scan was available for analysis or if the cross-sectional area of interest was not in the field of view.

Sagittal images of CT scans were selected at the level of lumbar-3 (L3) from the same contrast series by an experienced radiologist (C.Y.N.). The third lumbar vertebra region contains the psoas, paraspinal (erector spinae, quadratus lumborum), and abdominal wall muscles (external and internal obliques, rectus abdominus, transversus abdominus).

The images were analyzed by a trained single observer (N.C.H.) using SliceOmatic V5.0 software (Tomovision, Montreal, QC, Canada). This software enables specific tissue demarcation using density thresholds by Hounsfield units (HU; Fig. 1). The observer was blinded to the patient's postoperative course and survival.

To determine skeletal muscle mass at the L3 level, the cross-sectional skeletal muscle surface $\left(\mathrm{cm}^{2}\right)$ was identified and quantified by $\mathrm{HU}$ thresholds of -29 to +150 . $^{1,3,7}$

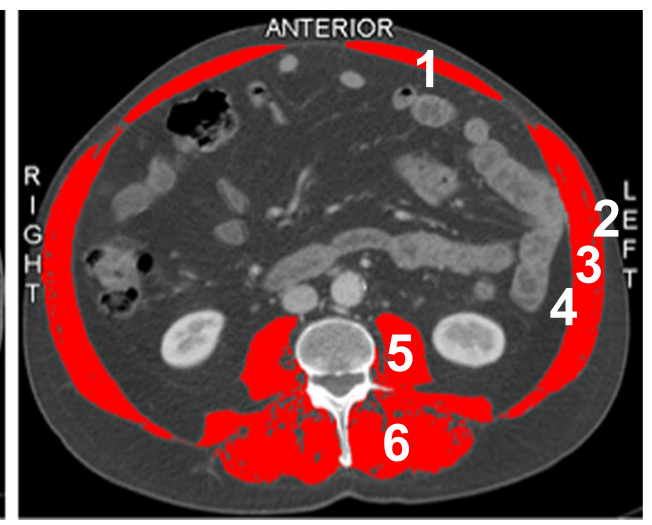

and a low MAI (MAI, 24.0). The skeletal muscle area is highlighted in red. 1 rectus abdominis, 2 external oblique, 3 internal oblique, 4 transverse abdominal, 5 psoas, 6 paraspinal 
Muscle area was normalized for height in meters squared $\left(\mathrm{m}^{2}\right)$ and reported as lumbar skeletal muscle index (SMI) $\left(\mathrm{cm}^{2} / \mathrm{m}^{2}\right)$. The muscle attenuation index (MAI) was determined by calculating the average HU at the L3 level. Intramuscular adipose tissue was additionally identified and quantified by $\mathrm{HU}$ thresholds of -190 to -30 .,13 $^{7,13}$

\section{Statistical Analysis}

To determine cutoff values for low SMI and MAI, we calculated sex-specific cutoff values by optimum stratification, as previously described., ${ }^{1,21,22}$ Optimum stratification is a statistical method that determines the threshold value of a continuous variable (SMI, MAI), which is based on log-rank statistics and best separates patients in terms of time to an event outcome (death).

Differences between groups were analysed using Pearson's chi-square test and the independent $t$ test as appropriate. Correlations between continuous variables were assessed using Pearson's correlation coefficients. All $p$ values were considered significant at the 0.05 level. Survival data are reported as median (range). OS rates, defined as the months of survival after PD, were calculated and compared using the Kaplan-Meier method. Conventional covariates included in the univariable survival analysis next to the dichotomous variables SMI and MAI were sex, age, American Society of Anesthesiologists (ASA) grade, tumor category, lymph node metastasis, tumor size, tumor grade, and resection margin status. ${ }^{16,23-27}$ The conventional covariates included in the univariate analysis for major postoperative complications next to SMI and MAI were sex, age, ASA grade, body mass index (BMI), diabetes mellitus, and tumor location. ${ }^{28}$ Characteristics with a $p$ value lower than 0.10 in the univariate analysis were entered into multivariable models. Statistical analyses were performed using IBM SPSS statistics version 21 (IBM, Armonk, NY, USA).

\section{RESULTS}

\section{Study Cohort}

The study identified 281 patients. Patients were excluded when a CT scan was inadequate to determine SMI or MAI $(n=97,35 \%)$ or when patient height could not be retrieved $(n=18,6 \%)$. Consequently, 166 patients were analyzed. The baseline characteristics between included and excluded patients did not differ (Supplementary Table 1). The baseline characteristics for the patients with low SMI and normal SMI, and for those with low MAI and normal SMA are shown in Table 1. Some significant differences are observed in the baseline characteristics. The median interval between the final preoperative $\mathrm{CT}$ scan and the date of surgery was 55 days [interquartile range (IQR), 39-74 days].

The sex-specific cutoff values for low SMI were 53.5 $\mathrm{cm}^{2} / \mathrm{m}^{2}$ for males and $46.4 \mathrm{~cm}^{2} / \mathrm{m}^{2}$ for females. Low SMI was present in 130 patients (78\%). The sex-specific cutoff values for low MAI were $36.3 \mathrm{HU}$ for males and $36.0 \mathrm{HU}$ for females. Low MAI was present in 81 patients (49\%). Low MAI was negatively correlated with intramuscular adipose tissue area $(r=-0.65 ; p<0.001)$.

\section{Overall Survival}

The median follow-up period for the patients who were alive on 1 September 2015 was 71 months. The median survival time for the patients receiving PD for periampullary carcinoma was 44 months (range 0-181 months). During the follow-up period, 91 patients (55\%) died.

The OS curves for the patients with low SMI and normal SMI and those with low MAI and normal SMI are shown in Fig. 2. The median OS for the patients with low SMI was 72 months, which did not differ from the median OS of 124 months for the patients with normal SMI $(p=0.28$; Fig. 2a). The median OS for the patients with low MAI was 39 months, significantly lower than the median OS of 98 months for the patients with normal MAI $(p<0.001$; Fig. 2b).

Low SMI was not associated with an increased hazard ratio (HR) of death [HR 1.3; $95 \%$ confidence interval (CI) 0.8-1.2]. Low MAI was associated with an increased hazard ratio of death (HR 2.4; $95 \%$ CI 1.6-3.8). Other factors associated with $\mathrm{OS}$ in the univariate analysis included age (HR 1.0; $95 \%$ CI 1.0-1.1), ASA score of 3 or 4 (HR 1.7; $95 \%$ CI 1.0-2.7), T3 tumor stage (HR 2.3; $95 \%$ CI 1.2-4.6), lymph node metastasis (HR 2.5; $95 \%$ CI 1.6-3.8), and microscopic radicality (HR 2.8; $95 \%$ CI $1.8-4.2)$.

In the multivariable analysis, low MAI remained independently associated with decreased OS (HR 1.95; $95 \%$ CI 1.2-3.3). The independent predictors of OS also included lymph node metastasis (HR 1.8; $95 \%$ CI 1.1-2.9) and microscopic radicality (HR 2.0; $95 \%$ CI $1.2-3.4$ ) (Table 2).

\section{Major Postoperative Complications}

The incidence of overall morbidity, major complications, and International Study Group of Pancreatic Surgery (ISGPS) complications is compared between patients with low and normal SMI and patients with low and normal MAI in Table 3. After PD, the overall morbidity rate was $66.3 \%$ (110 patients). The overall morbidity rate was similar between the patients with low SMI $(66.9 \%)$ and 


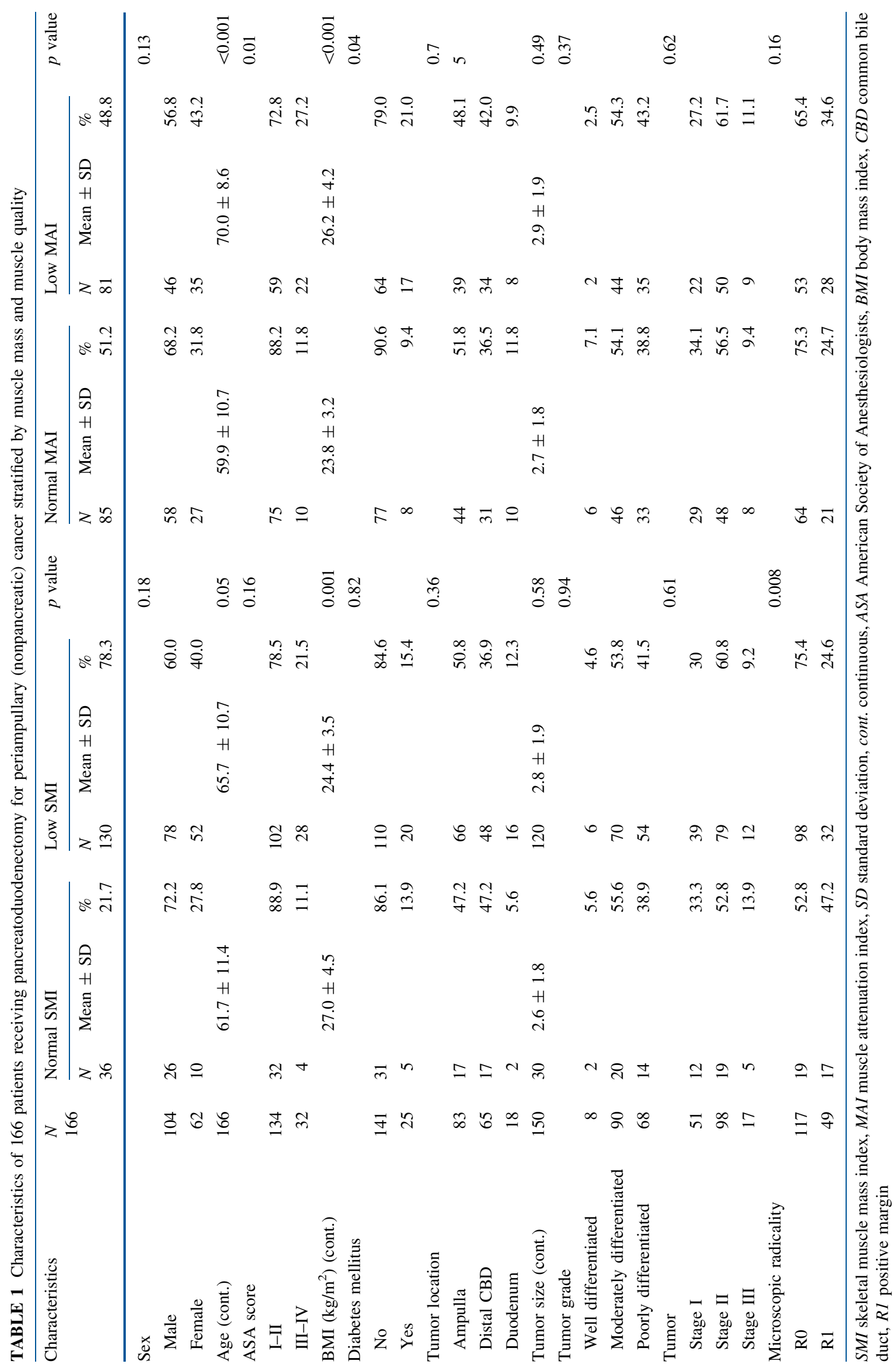



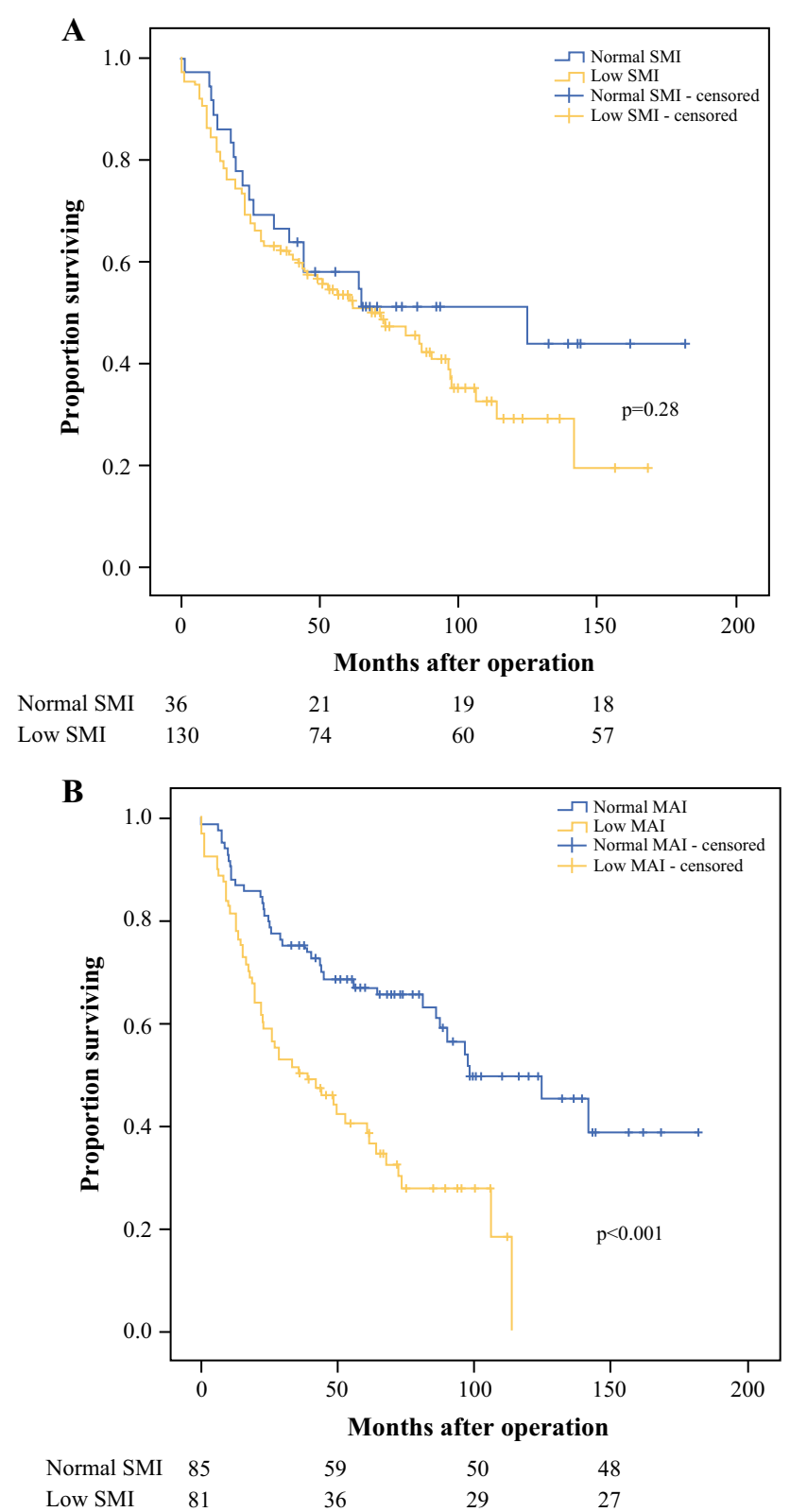

FIG. 2 Overall survival rates after pancreatoduodenectomy for periampullary, nonpancreatic cancer according to a skeletal muscle index and $\mathbf{b}$ skeletal muscle attenuation

those with normal SMI $(63.9 \%)(p=0.73)$. The overall morbidity was significantly higher for the patients with low MAI $(75.3 \%)$ than for the patients with normal MAI $(57.6 \%)(p=0.02)$.

The incidence of major complications was $47 \%$ (78 patients). The incidence of major complications was statistically equal between the patients with low SMI (47.7 \%) and those with normal SMI $(44.4 \%)(p=0.73)$. The incidence of major complications was significantly higher for the patients with low MAI (58 \%) than for the patients with normal MAI $(36.5 \%)(p=0.005)$. The patients with low SMI and those with normal SMI had similar rates for grades B and C postoperative pancreatic fistula (25.0 vs $29.2 \% ; p=0.62$ ), grades $\mathrm{B}$ and $\mathrm{C}$ postpancreatectomy hemorrhage (5.6 vs $6.9 \% ; p=0.77)$, and grades $\mathrm{B}$ and $\mathrm{C}$ delayed gastric emptying ( 38.9 vs $37.7 \% ; p=0.97$ ). The patients with low MAI experienced more grade $\mathrm{B}$ or $\mathrm{C}$ postpancreatectomy hemorrhage (11.1 vs $2.4 \% ; p=0.02)$ and more grade $\mathrm{B}$ or $\mathrm{C}$ delayed gastric emptying (46.9 vs $29.4 \% ; p=0.03)$. The rate for grades $\mathrm{B}$ and $\mathrm{C}$ postoperative pancreatic fistula was similar between the patients with low and high MAI (32.1 vs $24.7 \%$; $p=0.29$ ).

Low MAI was associated with an increased hazard ratio of major postoperative complications (odds ratio [OR] 2.4; $95 \%$ CI 1.3-4.5) but low SMI was not (OR 1.1; $95 \%$ CI $0.5-2.4)$. The only other factor associated with major complications in the univariate analysis was BMI (OR 1.1; $95 \%$ CI 1.0-1.2). After adjustment for potential confounding factors, only low MAI retained an independent association with the occurrence of major postoperative complications (OR 1.9; $95 \%$ CI 1.0-3.8) (Table 4).

\section{DISCUSSION}

In this first study investigating CT-derived body composition measures in patients undergoing $\mathrm{PD}$ for periampullary, nonpancreatic cancer, low muscle quality, but not low muscle mass, was independently associated with decreased survival and an increased incidence of major postoperative complications.

Various well-established patient and tumor characteristics are known to affect short- and long-term postoperative outcomes for patients with pancreatic and periampullary cancer. ${ }^{16,23-26}$ Our study adds to the previously identified risk factors for these patients, with a prognostic value equalling that of other conventional covariates.

The recent international interest in CT-derived body composition measures has led to identification of muscle mass and muscle quality as new independent predictors of outcome, with growing evidence to validate their prognostic impact. ${ }^{2}$ After some very recent studies investigating pancreatic cancer, ${ }^{3-6,10}$ our study is a first step toward determining the use of preoperative CT-derived body composition measures to stratify all patients receiving PD for cancer into risk categories and to assist in shared decision making.

Ideally, treatment of low muscle quality would lead to improved postoperative outcomes. Unfortunately, no such treatment is currently available. ${ }^{1}$ A randomized controlled trial with 52 nonsurgical, elderly patients demonstrated the clear effects of moderate physical exercise on age-related decline in muscle strength, but it involved a 12-month training program of moderate physical activity ${ }^{29}$ and 
TABLE 2 Multivariable Cox regression analysis of overall survival after pancreatoduodenectomy for periampullary, nonpancreatic cancer

\begin{tabular}{|c|c|c|c|c|c|c|c|}
\hline \multirow[t]{2}{*}{ Characteristics } & \multirow{2}{*}{$\begin{array}{l}N \\
166\end{array}$} & \multicolumn{3}{|c|}{ Univariable } & \multicolumn{3}{|c|}{ Multivariable } \\
\hline & & HR & $95 \% \mathrm{CI}$ & $p$ value & HR & $95 \% \mathrm{CI}$ & $p$ value \\
\hline Female sex & 62 & 0.70 & $0.46-1.05$ & 0.09 & 0.71 & $0.46-1.10$ & 0.13 \\
\hline Age (cont.) & 166 & 1.03 & $1.01-1.05$ & 0.003 & 1.01 & $0.98-1.03$ & 0.52 \\
\hline ASA score 3-4 & 32 & 1.65 & $1.01-2.71$ & 0.05 & 1.22 & $0.73-2.05$ & 0.45 \\
\hline \multicolumn{8}{|l|}{ Tumor category } \\
\hline $\mathrm{T} 1$ & & Ref & - & & Ref & & \\
\hline $\mathrm{T} 2$ & & 1.22 & $0.58-2.58$ & 0.60 & 0.85 & $0.39-1.83$ & 0.68 \\
\hline $\mathrm{T} 3$ & & 2.34 & $1.18-4.64$ & 0.02 & 1.21 & $0.57-2.59$ & 0.63 \\
\hline $\mathrm{T} 4$ & & 2.12 & $0.88-5.13$ & 0.10 & 0.89 & $0.34-2.32$ & 0.80 \\
\hline Lymph node metastasis $^{\mathrm{a}}$ & & 2.48 & $1.63-3.77$ & $<0.001$ & 1.82 & $1.14-2.91$ & 0.01 \\
\hline Tumor size & & 1.03 & $0.92-1.15$ & 0.58 & & & \\
\hline \multicolumn{8}{|l|}{ Tumor grade } \\
\hline Well differentiated & 8 & Ref & - & & & & \\
\hline Moderately differentiated & 90 & 1.57 & $0.49-5.09$ & 0.45 & 1.29 & $0.37-4.47$ & 0.69 \\
\hline Poorly differentiated & 68 & 2.75 & $0.85-8.91$ & 0.09 & 1.80 & $0.52-6.21$ & 0.35 \\
\hline Microscopic irradicality (R1) & 49 & 2.75 & $1.80-4.23$ & $<0.001$ & 2.01 & $1.20-3.36$ & 0.01 \\
\hline Low SMI & 130 & 1.33 & $0.79-1.24$ & 0.28 & & & \\
\hline Low MAI & 81 & 2.44 & $1.58-3.77$ & $<0.001$ & 1.95 & $1.16-3.29$ & 0.01 \\
\hline
\end{tabular}

$H R$ hazard ratio, $C I$ confidence interval, cont. continuous, ASA American Society of Aaesthesiologists, $R 1$ positive margin, SMI skeletal muscle mass index, MAI muscle attenuation index

a There were no patients with distant metastasis

TABLE 3 Complications after 166 pancreatoduodenectomies for periampullary, nonpancreatic cancer

\begin{tabular}{|c|c|c|c|c|c|c|}
\hline & $\begin{array}{l}\text { Normal SMI } \\
(n=36) n(\%)\end{array}$ & $\begin{array}{l}\text { Low SMI } \\
(n=130) n(\%)\end{array}$ & $p$ value & $\begin{array}{l}\text { Normal MAI } \\
(n=85) n(\%)\end{array}$ & $\begin{array}{l}\text { Low MAI } \\
(n=81) n(\%)\end{array}$ & $p$ value \\
\hline Overall morbidity & $23(63.9 \%)$ & $87(66.9 \%)$ & 0.73 & $49(57.6 \%)$ & $61(75.3 \%)$ & 0.02 \\
\hline Major complication $^{\mathrm{a}}$ & $16(44.4 \%)$ & $62(47.7 \%)$ & 0.73 & $31(36.5 \%)$ & $47(58.0)$ & 0.005 \\
\hline Postoperative fistula grades B \& C & $9(25 \%)$ & $38(29.2 \%)$ & 0.62 & $21(24.7 \%)$ & $26(32.1 \%)$ & 0.29 \\
\hline Postpancreatectomy hemorrhage grades B \& C & $2(5.6 \%)$ & $9(6.9 \%)$ & 0.77 & $2(2.4 \%)$ & $9(11.1 \%)$ & 0.02 \\
\hline Delayed gastric emptying grades B \& C & $14(38.9 \%)$ & $49(37.7 \%)$ & 0.97 & $25(29.4 \%)$ & $38(46.9 \%)$ & 0.03 \\
\hline
\end{tabular}

SMI skeletal muscle mass index, MAI muscle attenuation index

a Clavien-Dindo grade 3 or higher complication

therefore has no relevance in the preoperative setting or during neoadjuvant treatment. However, several prehabilitation strategies consisting of physical exercise have demonstrated improved postoperative outcomes. ${ }^{30}$ The effect of preoperative muscle or total-body exercises on postoperative outcomes for patients with low muscle mass or low muscle quality should be subject to future investigation.

Until recently, very little research on skeletal muscle attenuation (quality) was conducted. Some recent studies have investigated the prognostic significance of low muscle attenuation for patients with pancreatic cancer. ${ }^{4,5,10}$ One study included 230 patients undergoing PD, distal pancreatectomy, or total pancreatectomy for pancreatic ductal adenocarcinoma and identified muscle attenuation as an independent predictor of both worse survival and recurrence-free survival. ${ }^{4}$ In another study of 104 pancreatoduodenectomies, skeletal muscle attenuation was an independent predictor of severe complications according to the National Surgical Quality Improvement Program (NSQIP). ${ }^{5}$ It was not described whether periampullary cancer in the final pathology diagnosis was included in this study. Recently, attenuation of the psoas muscle on preoperative CT scan also was identified as an independent predictor of 1-year mortality for 518 elderly patients receiving HPB surgery, including pancreatic resection. ${ }^{10}$ In 
TABLE 4 Uni- and multivariate regression analyses of major postoperative morbidity after pancreatoduodenectomy for periampullary, nonpancreatic cancer

\begin{tabular}{|c|c|c|c|c|c|c|c|}
\hline \multirow[t]{2}{*}{ Characteristics } & \multirow{2}{*}{$\begin{array}{l}N \\
166\end{array}$} & \multicolumn{3}{|c|}{ Univariable } & \multicolumn{3}{|c|}{ Multivariable } \\
\hline & & OR & $95 \% \mathrm{CI}$ & $p$ value & OR & $95 \%$ CI & $p$ value \\
\hline Female sex & 62 & 0.99 & $0.53-1.85$ & 0.97 & & & \\
\hline Age (cont.) & 166 & 1.02 & $0.99-1.05$ & 0.12 & & & \\
\hline ASA score 3-4 & 32 & 0.85 & $0.39-1.85$ & 0.68 & & & \\
\hline BMI $\left(\mathrm{kg} / \mathrm{m}^{2}\right)$ (cont.) & 166 & 1.10 & $1.01-1.20$ & 0.03 & 1.07 & $0.98-1.17$ & 0.13 \\
\hline Diabetes mellitus & 25 & 0.87 & $0.37-2.04$ & 0.75 & & & \\
\hline \multicolumn{8}{|l|}{ Tumor location } \\
\hline Ampulla & 83 & Ref & - & & & & \\
\hline Distal bile duct & 65 & 0.91 & $0.47-1.74$ & 0.77 & & & \\
\hline Duodenum & 18 & 1.41 & $0.51-3.93$ & 0.51 & & & \\
\hline Low SMI & 130 & 1.14 & $0.54-2.39$ & 0.73 & & & \\
\hline Low MAI & 81 & 2.41 & $1.29-4.50$ & 0.006 & 1.93 & $1.01-3.77$ & 0.049 \\
\hline
\end{tabular}

OR odds ratio, $C I$ confidence interval, cont. continuous, ASA American Society of Anesthesiologists, BMI body mass index, SMI skeletal muscle mass index, MAI muscle attenuation index

the limited number of studies available, skeletal muscle quality was an important covariate in the prognosis of patients receiving PD for cancer.

Previous studies have identified muscle mass as an independent predictor of postoperative complications and survival and also after resection of pancreatic cancer. ${ }^{1-6}$ In one of the earlier, large series after resection of pancreatic adenocarcinoma, muscle mass was not associated with the risk of overall morbidity or serious complications but was associated with an increased risk of 3-year mortality. ${ }^{3}$ To our surprise, we did not find an association between muscle mass and postoperative major complications or survival in the univariate analysis. It is unlikely that the influence of muscle mass on postoperative outcomes is different after resection of periampullary cancer compared with pancreatic cancer, although the incidence of complications does differ between the two groups. ${ }^{28,31,32}$ It is unclear whether the unequal distribution of patients between low muscle mass $(78 \%)$ and normal muscle mass $(22 \%)$ could be a reason for this finding.

We used the preferred and accepted method (optimal stratification) to find the most significant cutoff for L3 muscle index, as previously described. ${ }^{1,21} \mathrm{We}$ found different cutoffs for men and women, which suggests that other, "static" cutoffs do not suit our cohort, so we believe that using these cutoffs would lead to inaccurate effect measurement. However, also when other previously used cutoff values for low muscle mass were applied, muscle mass still showed no effect on survival. ${ }^{2}$ The patients were better distributed among the muscle quality categories (51 vs $49 \%$ ). This suggests that some patients had a low muscle mass, but with normal muscle quality.
We found a significant negative correlation between MAI and intramuscular adipose tissue on preoperative CT scan, which reflects an accumulation of intramuscular lipid content. ${ }^{11-13}$ A higher muscle lipid content has been related to loss of muscle strength. ${ }^{14}$ This may explain why muscle quality and not muscle mass was an independent predictor of survival and major postoperative complications.

Complications after pancreatic surgery are very common. ${ }^{17,19,20}$ After resection for periampullary cancer, complications may occur even more frequently than after resection for pancreatic adenocarcinoma. ${ }^{28,31,32}$ The incidence of overall and major morbidity did not differ between the patients with low and normal muscle mass. The patients with low muscle quality experienced overall and major complications more frequently than the patients with normal muscle quality. These findings are reflected in our identification of low muscle quality as an independent predictor of survival and major complications.

We used several of the strongest and most frequent covariates predicting postoperative outcomes after pancreatic cancer surgery. ${ }^{16,23-26}$ Unfortunately, the presence of perineural or perivascular invasion was documented only incidentally by the pathologist and could therefore not be incorporated into our analysis.

We found a relatively long interval (median 55 days) between the final preoperative CT scan and the date of surgery. Possibly, as patients are referred to our tertiary hospital including CT scan, the interval is increased due to first discussion of the patient within a multidisciplinary team meeting, after which the operation is planned. Findings have shown that scans performed at a longer interval before surgery increases the incidence of intraoperative 
metastatic disease. ${ }^{33}$ Patient and event numbers were unfortunately too small to determine whether results would be different if patients who had a scan performed more than 30 days before the surgery were excluded.

This study had some limitations. We had to exclude a relatively high number of patients due to inadequacy of $\mathrm{CT}$ scans. However, the baseline characteristics did not differ significantly between the included and excluded patients (Supplementary Table 1). Furthermore, we identified some differences in baseline characteristics, mainly, a lower R1 rate for the patients with a low muscle mass and a higher BMI for the patients with low muscle attenuation than for the other patients. However, we corrected for these and other possible confounders in our analyses. Finally, we included three periampullary cancer types in our cohort. Survival and the incidence of complications may differ between the three types of periampullary tumors (duodenum, papilla, and distal bile duct tumors). ${ }^{16,28}$ However, in the sensitivity analysis, including also the cancer type in the multivariable analyses did not influence survival or the rate of major complications (data not shown).

\section{CONCLUSION}

In a large cohort of patients undergoing PD for periampullary, nonpancreatic cancer, low muscle quality, but not low muscle mass, predicted poor survival and major postoperative complications. Preoperative CT scans contain valuable information on patient body composition that may improve preoperative risk assessment and can support shared decision making.

ACKNOWLEDGMENT This research was funded in part by a grant from the Dutch Cancer Society (Grant No. UVA2013-5842).

OPEN ACCESS This article is distributed under the terms of the Creative Commons Attribution 4.0 International License (http:// creativecommons.org/licenses/by/4.0/), which permits unrestricted use, distribution, and reproduction in any medium, provided you give appropriate credit to the original author(s) and the source, provide a link to the Creative Commons license, and indicate if changes were made.

\section{REFERENCES}

1. Prado CM, Lieffers JR, McCargar LJ, et al. Prevalence and clinical implications of sarcopenic obesity in patients with solid tumours of the respiratory and gastrointestinal tracts: a population-based study. Lancet Oncol. 2008;9:629-35.

2. Levolger S, van Vugt JL, de Bruin RW, JN IJ. Systematic review of sarcopenia in patients operated on for gastrointestinal and hepatopancreatobiliary malignancies. $B r \quad J$ Surg. 2015;102: 1448-58.

3. Peng P, Hyder O, Firoozmand A, et al. Impact of sarcopenia on outcomes following resection of pancreatic adenocarcinoma. $J$ Gastrointest Surg. 2012;16:1478-86.
4. Okumura S, Kaido T, Hamaguchi Y, et al. Impact of preoperative quality as well as quantity of skeletal muscle on survival after resection of pancreatic cancer. Surgery. 2015;157:1088-98.

5. Sur MD, Namm JP, Hemmerich JA, Buschmann MM, Roggin KK, Dale W. Radiographic sarcopenia and self-reported exhaustion independently predict NSQIP serious complications after pancreaticoduodenectomy in older adults. Ann Surg Oncol. 2015;22:3897-904.

6. Amini N, Spolverato G, Gupta R, et al. Impact total psoas volume on short- and long-term outcomes in patients undergoing curative resection for pancreatic adenocarcinoma: a new tool to assess sarcopenia. J Gastrointest Surg. 2015;19:1593-602.

7. Martin L, Birdsell L, Macdonald N, et al. Cancer cachexia in the age of obesity: skeletal muscle depletion is a powerful prognostic factor, independent of body mass index. J Clin Oncol. 2013;31:1539-47.

8. Sabel MS, Lee J, Cai S, Englesbe MJ, Holcombe S, Wang S. Sarcopenia as a prognostic factor among patients with stage III melanoma. Ann Surg Oncol. 2011;18:3579-85.

9. Antoun S, Lanoy E, Iacovelli R, et al. Skeletal muscle density predicts prognosis in patients with metastatic renal cell carcinoma treated with targeted therapies. Cancer. 2013;119:3377-84.

10. Wagner D, Buttner S, Kim Y, et al. Clinical and morphometric parameters of frailty for prediction of mortality following hepatopancreaticobiliary surgery in the elderly. $\mathrm{Br} J$ Surg. 2016;103:e83-92.

11. Goodpaster BH, Park SW, Harris TB, et al. The loss of skeletal muscle strength, mass, and quality in older adults: the health, aging, and body composition study. J Gerontol A Biol Sci Med Sci. 2006;61:1059-64.

12. Newman AB, Kupelian V, Visser M, et al. Strength, but not muscle mass, is associated with mortality in the health, aging, and body composition study cohort. J Gerontol A Biol Sci Med Sci. 2006;61:72-7.

13. Aubrey J, Esfandiari N, Baracos VE, et al. Measurement of skeletal muscle radiation attenuation and basis of its biological variation. Acta Physiol Oxford. 2014;210:489-97.

14. Marcus RL, Addison O, Kidde JP, Dibble LE, Lastayo PC. Skeletal muscle fat infiltration: impact of age, inactivity, and exercise. J Nutr Health Aging. 2010;14:362-6.

15. Cameron JL, He J. Two thousand consecutive pancreaticoduodenectomies. J Am Coll Surg. 2015;220:530-6.

16. Tol JA, Brosens LA, van Dieren S, et al. Impact of lymph node ratio on survival in patients with pancreatic and periampullary cancer. Br J Surg. 2015;102:237-45.

17. Bassi C, Dervenis C, Butturini G, et al. Postoperative pancreatic fistula: an international study group (ISGPF) definition. Surgery. 2005;138:8-13.

18. Clavien PA, Barkun J, de Oliveira ML, et al. The Clavien-Dindo classification of surgical complications: five-year experience. Ann Surg. 2009;250:187-96.

19. Wente MN, Bassi C, Dervenis C, et al. Delayed gastric emptying (DGE) after pancreatic surgery: a suggested definition by the International Study Group of Pancreatic Surgery (ISGPS). Surgery. 2007;142:761-8.

20. Wente MN, Veit JA, Bassi C, et al. Postpancreatectomy hemorrhage (PPH): an International Study Group of Pancreatic Surgery (ISGPS) definition. Surgery. 2007;142:20-5.

21. van Vledder MG, Levolger S, Ayez N, Verhoef C, Tran TC, Ijzermans JN. Body composition and outcome in patients undergoing resection of colorectal liver metastases. Br J Surg. 2012;99:550-7.

22. Coelen RJ, Wiggers JK, Nio CY, et al. Preoperative computed tomography assessment of skeletal muscle mass is valuable in predicting outcomes following hepatectomy for perihilar cholangiocarcinoma. HPB Oxford. 2015;17:520-8. 
23. Paniccia A, Hosokawa P, Henderson W, et al. Characteristics of 10-year survivors of pancreatic ductal adenocarcinoma. JAMA Surg. 2015;150:701-10.

24. Bouvet M, Gamagami RA, Gilpin EA, et al. Factors influencing survival after resection for periampullary neoplasms. Am J Surg. 2000;180:13-7.

25. Yeo CJ, Cameron JL, Lillemoe KD, et al. Pancreaticoduodenectomy for cancer of the head of the pancreas: 201 patients. Ann Surg. 1995;221:721-31

26. Nitecki SS, Sarr MG, Colby TV, van Heerden JA. Long-term survival after resection for ductal adenocarcinoma of the pancreas: is it really improving? Ann Surg. 1995;221:59-66.

27. Hatzaras I, George N, Muscarella P, Melvin WS, Ellison EC, Bloomston M. Predictors of survival in periampullary cancers following pancreaticoduodenectomy. Ann Surg Oncol. 2010;17:991-7.

28. Roberts KJ, Sutcliffe RP, Marudanayagam R, et al. Scoring system to predict pancreatic fistula after pancreaticoduodenectomy: a UK multicenter study. Ann Surg. 2015;261:1191-7.
29. Goodpaster BH, Chomentowski P, Ward BK, et al. Effects of physical activity on strength and skeletal muscle fat infiltration in older adults: a randomized controlled trial. J Appl Physiol. (1985) 2008;105:1498-503.

30. Santa Mina D, Clarke H, Ritvo P, et al. Effect of total-body prehabilitation on postoperative outcomes: a systematic review and meta-analysis. Physiotherapy. 2014;100:196-207.

31. Callery MP, Pratt WB, Kent TS, Chaikof EL, Vollmer CM Jr. A prospectively validated clinical risk score accurately predicts pancreatic fistula after pancreatoduodenectomy. J Am Coll Surg. 2013;216:1-14.

32. Miller BC, Christein JD, Behrman SW, et al. A multi-institutional external validation of the fistula risk score for pancreatoduodenectomy. J Gastrointest Surg. 2014;18:172-79.

33. Glant JA, Waters JA, House MG, et al. Does the interval from imaging to operation affect the rate of unanticipated metastasis encountered during operation for pancreatic adenocarcinoma? Surgery. 2011;150:607-16. 